\title{
Application of Robust Control Design Techniques to the Aeroservoelastic Design Optimization of a Very Flexible UAV Wing
}

\author{
Sohrab Haghighat*, Hugh H.T. Liu ${ }^{\dagger}$ \\ University of Toronto Institute for Aerospace Studies, Toronto, Ontario, M3H 5T6, Canada \\ and \\ Joaquim R. R. A. Martins ${ }^{\ddagger}$ \\ Department of Aerospace Engineering, University of Michigan, 1320 Beal Avenue, Ann Arbor, MI
}

\begin{abstract}
A multidisciplinary design optimization framework is developed to integrate control system design and aerostructural design of an aircraft in the conceptual design phase. The aircraft's wings are very flexible and the aircraft's aeroservoelastic equations of motion are derived and integrated with the aerostructural analysis and control system design. A two degree of freedom multi-objective control architecture capable of structural load alleviation is proposed. The linear matrix inequalities technique is used to express the control design process as a convex optimization problem. Time-domain analysis of the aircraft encountering an atmospheric gust is included in the design process. The optimal trade-off between aerodynamics, structures and control systems is found by maximizing the endurance subjected to stress constraints.
\end{abstract}

\section{Introduction}

Over the past few decades, with the advancements in control technology, active control technology became a solution to many aerospace design problems, including aeroservoelastic problems. Based on the accumulated knowledge and experience, it has been well established that aeroservoelastic analysis and synthesis are not just endeavors of choice, but are an integral part of modern aircraft design. ${ }^{1]}$ The significance of aeroservoelastic considerations is even more evident in flexible aircraft design. The demand for long endurance aircraft, especially in unmanned aircraft application, has resulted in high aspect ratio configurations with very flexible wings, such as NASA's Helios. This added flexibility has drawn more interest toward the integration of aeroservoelastic synthesis in the aircraft design process.

While much research has been conducted on the application of active aeroelastic wing (AAW) technology to flutter suppression and wing load alleviation, $\sqrt{2 / 6]}$ the integration of aeroservoelastic considerations with other disciplines in the early stages of the design process has not been fully addressed. One of the earliest studies in aeroservoelastic optimization was conducted by Suzuki,$\frac{3}{3}$ who performed simultaneous structural and control design to minimize the wing structural weight. The optimization was subjected to control stability and stress constraints while flying through atmospheric gust. Idan et al. ${ }^{7}$ proposed an aeroservoelastic design framework where the interaction between the aircraft structure and control system was considered. First, a preliminary structural and control optimization was performed separately. The resulting structure and control systems were then used to perform a simultaneous optimization addressing closed-loop control margins and flutter. The same framework was later used to perform simultaneous structural and control optimization in the presence of parameter uncertainty ${ }^{[8}$ Zink et al.$^{9}$ addressed the design of structural parameters and gear ratios of a lightweight fighter performing symmetric and antisymmetric rolling maneuvers. The optimization formulation was based on static aeroelastic equations. The integrated approach results were compared to the results of a sequential method. The former was shown to be more effective and was converged to a lower structural weight. The same authors also considered maneuver load inaccuracies and its effects on the optimum design! 10

*PhD Candidate; sohrab.haghighat @ utoronto.ca, University of Toronto Institute for Aerospace Studies, AIAA Member.

$\dagger$ Associate Professor; liu@ utias.utoronto.ca, University of Toronto Institute for Aerospace Studies, AIAA Senior Member.

¥Associate Professor; jrram@ umich.edu, Department of Aerospace Engineering, University of Michigan, AIAA Senior Member. 


\section{A. Motivation}

Traditionally, the main focus of aeroservoelastic synthesis has been either to achieve the required maneuverability, or to avoid structural failure without having excessively stiffened wings. Only a few researchers have integrated planform and control system design in order to improve the overall aircraft performance metrics such as range, endurance, fuel consumption, and total emissions ${ }^{4}$ Investigations have shown that aircraft performance improvement is achievable by including control system design in the conceptual design process ${ }^{11 \mid 12}$ Also, various published results have shown that the simultaneous optimization results are superior to designs obtained through performing sequential optimization.9:13]14 This shows that further gains for active aeroservoelastic aircraft can be achieved if simultaneous optimization methods are used at the earlier stages of the airplane design process.

The exclusion of flight dynamics and control disciplines from the MDO design cycle not only results in a suboptimal solution, but may also have a detrimental effect on the design when highly flexible aircraft are considered. In such a case, active control systems and load alleviation techniques can alter the configuration dramatically, improving both the aircraft performance and the stability margin of the flexible aircraft. Highly flexible aircraft can deform in unexpected ways, depending on the atmospheric conditions, as in the case of the Helios aircraft incident $\frac{15}{15}$ Among the technical recommendations included in the accident investigation that followed the crash, the development of more advanced multidisciplinary techniques that include control systems and time-domain analysis methods appropriate to highly flexible structures was strongly emphasized. ${ }^{[15}$

The current work is a first step toward the inclusion of control systems design in the multidisciplinary design framework for a highly flexible aircraft considering time dependent maneuvers and gust excitations. In contrast to the current trend, where the aircraft structure is designed to withstand the critical loading conditions of a steady maneuvering flight, the maximum instantaneous stress is considered here. The maximum instantaneous stress is caused by performing maneuvers, flying through atmospheric turbulence, or both. The inclusion of the time-history analysis in the design cycle is expected to guide the optimizer away from the configurations that are more vulnerable to instabilities. In order to achieve this goal, the derivation of the equations of motion is performed in a way that captures the inertial and aerodynamic interactions between the rigid-body and deformable dynamics. Simpler formulations of the nonlinear equations of motion of a very flexible aircraft based on quasi-coordinate method had been developed and were presented in authors' previous publications. ${ }^{16}[18$ The complete derivation of the equations accounting for larger displacements and closer interactions between the two dynamics is presented in this paper. These equations allow for large rigid-body motions with structural deflections which are presented in a modal form.

\section{B. Proposed optimization framework}

The main goal of this work is to study the effect of optimizing the control system concurrently with the aircraft wing planform and structural sizing. The proposed framework consists of a system-level optimizer that iterates over the system-level design variables to maximize endurance, while satisfying stress constraints.

The application of model predictive control (MPC) techniques and linear quadratic regulators (LQR) has been used in previous work by the authors to develop gust and maneuver load alleviation systems 16$] 17$ Also, the results of integrated structural and active control system design optimization have been studied ${ }^{[18}$ In this work, a more advanced control architecture that is capable of simultaneously handling the rigid-body and the deformable dynamics is presented. This new, two degree of freedom control architecture is formulated in a linear matrix inequality (LMI) form and linked to a discipline-level convex optimizer. This control structure is specifically developed to address multiobjective control problems. The discipline-level objective function is a linear combination of frequency weighted rigid-body errors, structural deformations, and control effort. The system-level optimizer governs the control synthesis by varying the relative weight of different terms in the control design objective function. Mixed-norm optimization is considered in this work. While the infinity-norm $\left(\|\cdot\|_{\infty}\right)$ of the sensitivity transfer function is a good measure of the system's stability and robustness, the generalized 2-norm $\left(\|\cdot\|_{g}\right)$ is a better representation of the maximum structural deflections and rather than bounding the structural energy, it can keep the peak amplitude of the structural loading below a desirable level! 19

As previously mentioned, system-level objective is to maximize the endurance. This is calculated based on the cruise performance. The maximum structural stresses computed for flying through a discrete vertical gust. An aug- 
mented Lagrangian particle swarm optimizer, ALPSO, 20 which is a non-gradient based optimizer is used to solve the system-level problem. The choice of a non-gradient based optimizer is due to the stress calculation, which is based on the time-history simulation of the aircraft encountering gust. An unstable (or marginally stable) controller can result in a diverging time solution that causes discontinuities in the constraint function, which gradient based optimizers do not handle well.

In the next section, the nonlinear equations of motion of a very flexible aircraft are derived. The aerostructural analysis and control synthesis are discussed in Sections III and IV Then, in Section V, the optimization framework is explained and finally, the obtained results are presented and discussed in detail.

\section{Aeroservoelastic Formulation}

The development of a mathematical model that can be used to integrate flight dynamics with other disciplines such as structures and aerodynamics is the key to developing the proposed framework. The main challenge is to develop an efficient mathematical model that represents the real system accurately. Some of the previous work that addressed the AAW design was based on quasi-steady flight models, where structural deflections were converged to steady-state conditions $9.21[22$ Those models cannot be used to address the instantaneous stress and the system's stability. Other early efforts have tried to obtain the flexible aircraft equations of motion by simply adding a series of second-order differential equations to a rigid-body model where each set of equations represents one structural mode ${ }^{23 / 24}$

Schmidt et al..$^{25 \mid 26}$ developed the equations of motion for a flexible aircraft using mean-axes method. The use of aerodynamic strip theory and the assumption that structural displacments are small make the formulation less appealing for our application. More rigorous formulations have been developed using the quasi-coordinate methods in which the axes system is fixed to a specific point of the undeformed aircraft, such as the undeformed center of gravity. ${ }^{27}, 30$

The nonlinear equations of motion of a very flexible aircraft derived in this section using a body-fixed axes system. We begin with the Lagrange equations for quasi-coordinates,

$$
\begin{array}{r}
\frac{\mathrm{d}}{\mathrm{d} t}\left(\frac{\partial L}{\partial \mathbf{V}_{c}}\right)+\tilde{\omega} \frac{\partial L}{\partial \mathbf{V}_{c}}-C_{b i} \frac{\partial L}{\partial \mathbf{R}}=\mathbf{F}, \\
\frac{\mathrm{d}}{\mathrm{d} t}\left(\frac{\partial L}{\partial \boldsymbol{\omega}}\right)+\tilde{V}_{c} \frac{\partial L}{\partial \mathbf{V}_{c}}+\tilde{\omega} \frac{\partial L}{\partial \boldsymbol{\omega}}-D^{T}-1 \frac{\partial L}{\partial \boldsymbol{\theta}}=\mathbf{M}, \\
\frac{\mathrm{d}}{\mathrm{d} t}\left(\frac{\partial \hat{L}}{\partial \mathbf{v}}\right)-\frac{\partial \hat{T}}{\partial \mathbf{u}}+\frac{\partial \hat{\mathcal{F}}}{\partial \mathbf{v}}+\mathcal{L} \mathbf{u}=\hat{U},
\end{array}
$$

where, $\mathcal{F}$ represents the Rayleigh's dissipation term and $(\hat{\bullet})$ represents the volume density of the energy terms. Later in the derivation, the dissipation term will be dropped, which results in a more conservative flutter speed estimation. The two transformation matrices, $C_{b i}$ and $D$, perform the transformation of the inertial velocities into the velocity vectors in the body-fixed coordinate system. Figure 1 shows a deformable aircraft with its original and deformed wing, as well as the associated axes systems. $\mathfrak{F}_{I}$ represents the inertial frame and $\mathfrak{F}_{B}$ the body-fixed frame, not necessarily connected to the center of mass. The absolute position and velocity of an infinitesimal mass element $\mathrm{d} m$ on the aircraft is given by,

$$
\begin{aligned}
& \mathbf{R}=\mathbf{R}_{c}+\mathbf{r}+\mathbf{u} \\
& \mathbf{V}=\dot{\mathbf{R}}=\mathbf{V}_{c}+\boldsymbol{\omega} \times(\mathbf{r}+\mathbf{u})+\dot{\mathbf{u}}
\end{aligned}
$$

The above vectors are graphically represented in Figure 1

The structural deformations can be expressed in a discrete finite element formulation or as a linear combination of mode shapes. A common choice for these mode shapes are the eigenvectors of the structural system. Although the application of the modal representation in dynamic response and stability analysis is widely practiced, static aeroelastic analysis is usually based on discrete finite-element methods. ${ }^{31}$ This is mainly due to the effect of lumped forces, such as the weight of external stores, which the modal representation does not capture adequately, unless large number of 


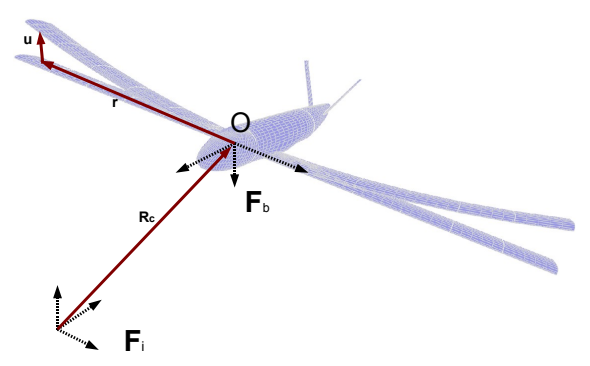

Figure 1. A schematic drawing of a deformed aircraft and the position vectors

modes (shape functions) are considered. In our case, since no such lumped forces are present on the aircraft wing, the modal representation is used for both static and dynamic analysis. The structural displacement and velocity can be expressed in the modal form are as follows,

$$
\mathbf{u}=\phi_{\mathbf{r}} \boldsymbol{\xi} \Rightarrow \dot{\mathbf{u}}=\phi_{\mathbf{r}} \dot{\boldsymbol{\xi}}
$$

where, $\boldsymbol{\xi}$ is a vector of modal amplitudes and $\phi_{\mathbf{r}}$ represents the modal deflection at location $\mathbf{r}$ and has the following form,

$$
\phi_{\mathbf{r}}=\left[\begin{array}{cccc}
x_{\mathbf{r}, 1} & x_{\mathbf{r}, 2} & \cdots & x_{\mathbf{r}, i} \\
y_{\mathbf{r}, 1} & y_{\mathbf{r}, 2} & \cdots & y_{\mathbf{r}, i} \\
z_{\mathbf{r}, 1} & z_{\mathbf{r}, 2} & \cdots & z_{\mathbf{r}, i}
\end{array}\right],
$$

where, $x_{\mathbf{r}, i}$ is the deformation at location $\mathbf{r}$ in the $x$ direction due to the $i^{\text {th }}$ structural shape function. Here, the gravitational force is modeled as a distributed force and therefore, the following terms in Equations (1) and (2) vanish. Thus, we can write,

$$
\frac{\partial L}{\partial \mathbf{R}}=0 \quad, \quad \frac{\partial L}{\partial \boldsymbol{\theta}}=0 .
$$

By using the structural shape functions, Equation (3) can be re-arranged and adapted to the modal form as follows,

$$
\frac{\mathrm{d}}{\mathrm{d} t}\left(\frac{\partial L}{\partial \dot{\boldsymbol{\xi}}}\right)-\frac{\partial T}{\partial \boldsymbol{\xi}}+\frac{\partial \mathcal{U}}{\partial \boldsymbol{\xi}}+\frac{\partial \mathcal{F}}{\partial \dot{\boldsymbol{\xi}}}=\mathbf{f}_{e},
$$

for which, $\mathcal{U}$ and $\mathcal{F}$ represent the strain energy and Rayleigh's dissipation for the complete aircraft structure. Also, $\mathbf{f}_{e}$ represents the modal forces that act on the aircraft structure.

\section{A. Kinetic Energy}

The kinetic energy of a deformable aircraft can be formulated as follows:

$$
\begin{aligned}
& T=\frac{1}{2} \int_{v} \rho \mathbf{V}^{T} \mathbf{V} \mathrm{d} v \\
& =\frac{1}{2} m \mathbf{V}_{c}^{T} \mathbf{V}_{c}+\mathbf{V}_{c}^{T}\left[\int_{v} \rho\left(\tilde{r}+\widetilde{\phi_{\mathbf{r}} \boldsymbol{\xi}}\right)^{T} \mathrm{~d} v\right] \boldsymbol{\omega}+\mathbf{V}_{c}^{T}\left[\int_{v} \rho \phi_{\mathbf{r}} \mathrm{d} v\right] \dot{\boldsymbol{\xi}}
\end{aligned}
$$

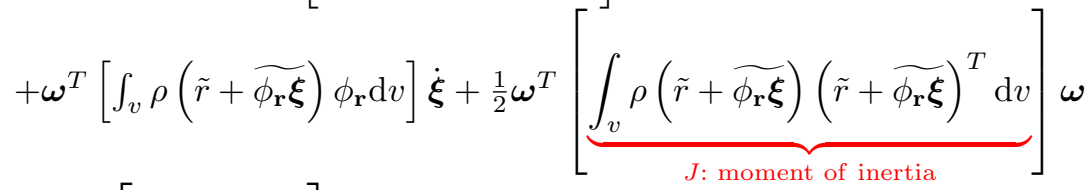

$$
\begin{aligned}
& +\frac{1}{2} \dot{\boldsymbol{\xi}}^{T}[\underbrace{\int_{v} \rho \phi_{\mathbf{r}}^{T} \phi_{\mathbf{r}} \mathrm{d} v}_{M_{e e}}] \dot{\boldsymbol{\xi}},
\end{aligned}
$$

where, $\sim$ represents the skew-symmetric matrix used for performing the cross-product. If the origin of $\mathfrak{F}_{B}$ is placed at the undeformed aircraft center of gravity, the term $\int_{v} \rho \tilde{r}^{T} \mathrm{~d} v$ is zero. 


\section{B. Potential Energy}

The strain energy of an elastic system can be written as,

$$
\mathcal{U}=\frac{1}{2} \mathbf{U}^{T} K_{G G} \mathbf{U}=\frac{1}{2} \boldsymbol{\xi}^{T} \Phi^{T} K \Phi \boldsymbol{\xi}=\frac{1}{2} \boldsymbol{\xi}^{T} K_{e e} \boldsymbol{\xi},
$$

where, $\Phi$ is the matrix of the shape functions for all nodes on the aircraft structure, which has the following form,

$$
\Phi=\left[\begin{array}{cccc}
x_{\mathbf{r}_{1}, 1} & x_{\mathbf{r}_{1}, 2} & \cdots & x_{\mathbf{r}_{1}, i} \\
y_{\mathbf{r}_{1}, 1} & y_{\mathbf{r}_{1}, 2} & \cdots & y_{\mathbf{r}_{1}, i} \\
z_{\mathbf{r}_{1}, 1} & z_{\mathbf{r}_{1}, 2} & \cdots & z_{\mathbf{r}_{1}, i} \\
x_{\mathbf{r}_{2}, 1} & x_{\mathbf{r}_{2}, 2} & \cdots & x_{\mathbf{r}_{2}, i} \\
y_{\mathbf{r}_{2}, 1} & y_{\mathbf{r}_{2}, 2} & \cdots & y_{\mathbf{r}_{2}, i} \\
z_{\mathbf{r}_{2}, 1} & z_{\mathbf{r}_{2}, 2} & \cdots & z_{\mathbf{r}_{2}, i} \\
\vdots & \vdots & & \vdots \\
x_{\mathbf{r}_{j}, 1} & x_{\mathbf{r}_{j}, 2} & \cdots & x_{\mathbf{r}_{j}, i} \\
y_{\mathbf{r}_{j}, 1} & y_{\mathbf{r}_{j}, 2} & \cdots & y_{\mathbf{r}_{j}, i} \\
z_{\mathbf{r}_{j}, 1} & z_{\mathbf{r}_{j}, 2} & \cdots & z_{\mathbf{r}_{j}, i}
\end{array}\right] .
$$

\section{Nonlinear Equations of Motion}

Substituting the kinetic and potential energy into Equations (1,2) and 8 ) and rearranging them, the equations of motion of a deformable aircraft become

$$
\begin{aligned}
& {\left[\begin{array}{ccc}
m \mathcal{I}_{3 \times 3} & X_{1}^{T} & S_{1} \\
X_{1} & J & S_{2}+X_{2} \\
S_{1}^{T} & S_{2}^{T}+X_{2}^{T} & M_{e e}
\end{array}\right]\left\{\begin{array}{l}
\dot{\mathbf{V}}_{c} \\
\dot{\omega} \\
\ddot{\xi}
\end{array}\right\}=} \\
& -\left[\begin{array}{ccc}
m \tilde{\omega} & \tilde{\omega} X_{1}^{T} & 2 \tilde{\omega} S_{1} \\
-\tilde{\omega} X_{1} & \tilde{\omega} J+\dot{J}+\tilde{V}_{c} X_{1} & \tilde{\omega}\left(S_{2}+X_{2}\right) \\
O_{n \times 3} & S_{1}^{T} V_{c}-2 \int_{v} \rho \phi_{\mathbf{r}}{ }^{T} \phi_{\mathbf{r}} \dot{\boldsymbol{\xi}} \mathrm{d} v+\int_{v} \rho \phi_{b l r}{ }^{T} \tilde{\omega} \tilde{r}^{T} \mathrm{~d} v & C_{e e}
\end{array}\right]\left\{\begin{array}{l}
\mathbf{V}_{c} \\
\boldsymbol{\omega} \\
\dot{\xi}
\end{array}\right\} \\
& -\left[\begin{array}{cc}
\mathcal{O}_{6 \times 6} & \mathcal{O}_{6 \times n} \\
\mathcal{O}_{n \times 6} & \int_{v} \rho \phi_{\mathbf{r}}^{T} \tilde{\omega}^{2} \phi_{\mathbf{r}} \mathrm{d} v+K_{e e}
\end{array}\right]\left\{\begin{array}{l}
\mathbf{R}_{c} \\
\boldsymbol{\theta} \\
\boldsymbol{\xi}
\end{array}\right\}+\left[\begin{array}{c}
m C_{b i} \\
\mathcal{O}_{3 \times 3} \\
S_{1}^{T} C_{b i}
\end{array}\right]\{\mathbf{g}\}+\left\{\begin{array}{c}
\mathbf{F} \\
\mathbf{M} \\
\mathbf{f}_{e}
\end{array}\right\}
\end{aligned}
$$

where, $\mathbf{F}$ and $\mathbf{M}$ are the total forces and moments about the origin of the body-frame, including propulsive and aerodynamic forces. $\mathbf{f}_{e}$ represents the generalized forces due to elastic deformations and is calculated as,

$$
\mathbf{f}_{e}=\int_{s} \phi_{r}^{T} \mathbf{P} d S
$$

where $\mathbf{P}$ is the pressure vector acting on the surface of the aircraft. Also, $S_{1}, S_{2}, X_{1}$ and $X_{2}$ are the rigid-elastic interaction terms and are defined as follows,

$$
\begin{aligned}
& S_{1}=\int_{v} \rho \phi_{r} \mathrm{~d} v, \\
& S_{2}=\int_{v} \rho \tilde{r} \phi_{r} \mathrm{~d} v, \\
& X_{1}=\int_{v} \rho \hat{\phi_{r} \boldsymbol{\xi}} \mathrm{d} v, \\
& X_{2}=\int_{v} \rho \hat{\phi_{r} \boldsymbol{\xi}} \phi_{r} \mathrm{~d} v .
\end{aligned}
$$

As can be seen from Equation (12), the above mentioned terms mainly appear in the inertia matrix and the force vector and create interaction between the rigid and deformable dynamics. As aircraft become more flexible, the relative magnitudes of there terms with respect to the other terms in Equation (12) become larger that results in a closer interaction between the two dynamics. 


\section{Aerostructural Analysis}

The aerostructural model used in this work is shown in Figure 2. The wing spar is modeled as a hollow tubular beam and the aerodynamic forces and moments are calculated using an in-house vortex lattice panel code.

Now that we have the equations of motion for a flexible aircraft, the steady-state equations can be derived. In order to derive the equations for rectilinear flight, we start from the equations of motion (12) and set all derivatives and $\boldsymbol{\omega}$ to zero, which yields,

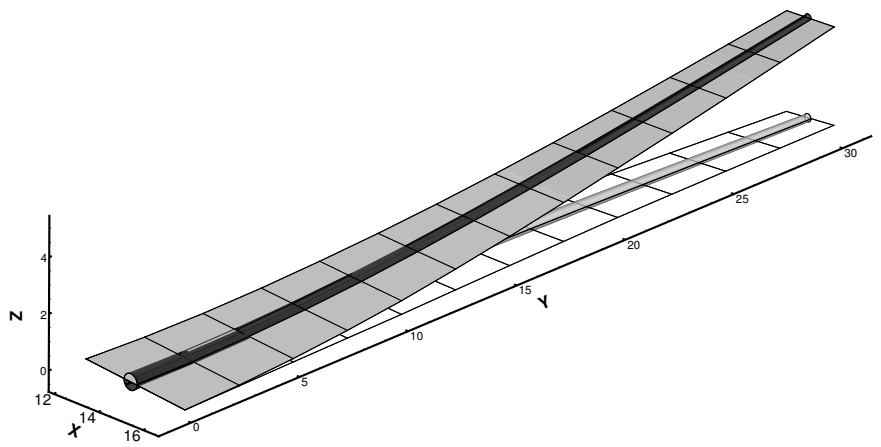

Figure 2. Aerostructural model showing the tubular spar and the wing panels

$$
\left\{\begin{array}{l}
\mathbf{F}+m C_{b i} \mathbf{g}=0 \\
\mathbf{M}=0 \\
-K_{e e} \boldsymbol{\xi}+\left(S_{1}\right)^{T} C_{b i} \mathbf{g}+\mathbf{f}_{e}=0
\end{array}\right.
$$

The above equations are coupled to each other through aerodynamic forces and moments, and can be used to solve the trim parameters $\left(C_{L}, C_{D}, \alpha, \delta_{e}\right)$ and the steady-state wing deformation $(\boldsymbol{\xi})$. Note that both the drag and the thrust forces are included in the force vector $(\mathbf{F})$ in the above equation. An iterative approach with successive-over-relaxation (SOR) is employed to find the steady-state flight conditions and deformations. This iteration is depicted in Figure 3 The term $d_{i}$ is the dynamic damping that is used to perform relaxation and its value changes dynamically for each cycle.

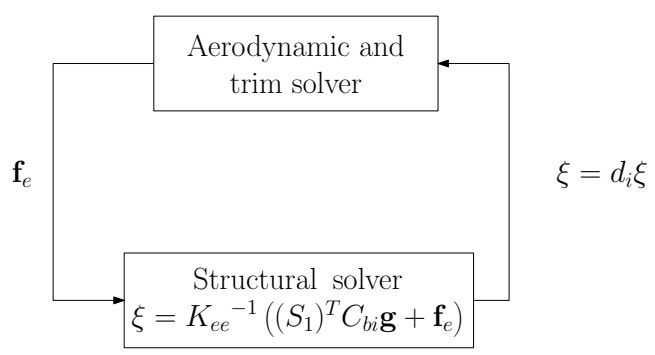

Figure 3. Aerostructural iterative solver

The angle of attack and the elevator deflection angle are adjusted to trim the aircraft for level flight. Lift and drag coefficients at cruise are used to calculate the aircraft endurance and the wing deflections are used to evaluate the structural stresses present in the constraints. The outputs of the aerostructural solver are used to linearize the equations of motion needed in the control synthesis. 


\section{A. Structural Modeling}

The aircraft fuselage and tail (horizontal and vertical) are considered to be rigid, and elastic deformations computed only for the aircraft wing. Grid finite elements - also known as torsional beams - are used to model the wing deformations. Each grid element has two nodes, each with three degrees of freedom: displacement in the $z$ direction and rotations about the $x$ and $y$ axes. The wing box is approximated as a circular cross section hollow tube with variable wall thickness along the span. The spar diameter is dictated by the airfoil chord and the thickness to chord ratio $(t / c)$, the spar thickness is selected by the optimizer as the structural sizing design variable. The stiffness and mass matrices for a grid element can be found in Paz and Leight. ${ }^{[32]}$ The von Mises stress can be calculated from the modal deflections. It is worth mentioning that, the c.g. line of the wing is not aligned with the elastic-axis of the wing. This factor has to be considered in development of the shape functions that are employed in the analysis and the shape functions have to represent the deformation of center of mass line.

\section{B. Aerodynamic Modeling}

The vortex lattice method (VLM) is used to calculate the aerodynamic forces and moments. Figure 4 shows the vortex rings and wake vortices generated for aerodynamic analysis of an aircraft configuration. The panel and wake vorticities are computed by enforcing the tangential flow condition at each panel. The vorticity of each panel is then used to compute the pressure on that panel. Using the pressure at each panel, rigid and elastic aerodynamic forces and moments can be computed as follows

$$
\begin{aligned}
& \mathbf{F}_{\text {aero }}=\sum_{j} \sum_{k} p_{j k} \hat{\mathbf{n}_{j k}} \Delta A_{j k}, \\
& \mathbf{M}_{a e r o}=\sum_{j} \sum_{k} \tilde{r}\left(p_{j k} \hat{\mathbf{n}_{j k}}\right) \Delta A_{j k}, \\
& \mathbf{f}_{e_{i}}=\left.\sum_{j} \sum_{k} \phi_{i}\right|_{j k} p_{j k} \hat{\mathbf{n}_{j k}} \Delta A_{j k},
\end{aligned}
$$

where $p_{j k}$ and $\hat{\mathbf{n}}_{j k}$ are the pressure and the normal vector for panel $j, k$, respectively. The deflection due to the $i^{t h}$ structural mode at panel $j, k$ is given by $\left.\phi_{i}\right|_{j k}$.

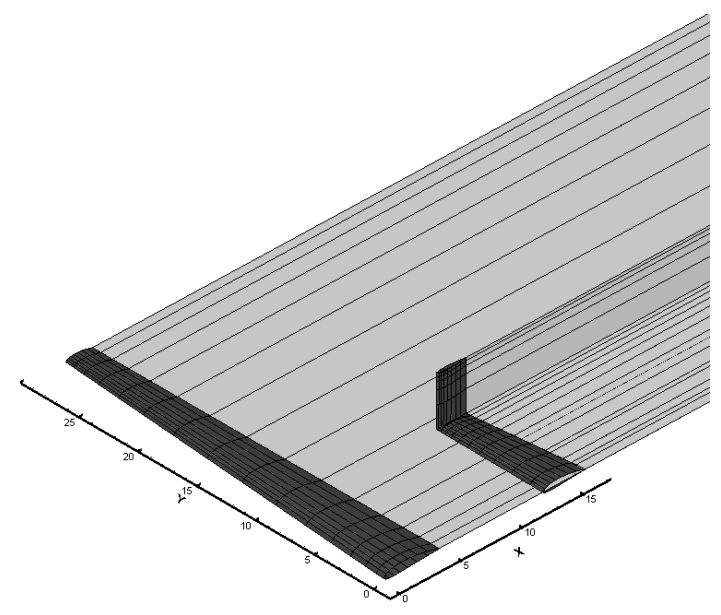

Figure 4. Sample meshed aircraft for aerodynamic analysis 


\section{Aeroservoelastic Control}

The first step in control system development is to extract the nominal plant representation. We also need to linearize the equations of motion. Substituting the perturbed flight parameters into Equation (12) and linearizing, yields,

$$
\begin{aligned}
& \left\{\begin{array}{l}
\delta \dot{\mathbf{V}}_{c} \\
\delta \dot{\boldsymbol{\omega}} \\
\delta \ddot{\boldsymbol{\xi}}
\end{array}\right\}=\mathbf{M}^{-1}\left(\left[\begin{array}{ccc}
\mathcal{O}_{3 \times 3} & m \tilde{V}_{c_{0}} & \mathcal{O}_{3 \times n} \\
\mathcal{O}_{3 \times 3} & X_{1_{0}}^{T} V_{c_{0}}-\tilde{V}_{c_{0}} X_{1_{0}} & \mathcal{O}_{3 \times n} \\
\mathcal{O}_{3 \times 3}
\end{array}\right]\left\{\begin{array}{l}
\delta \mathbf{V}_{c} \\
\delta \boldsymbol{\omega} \\
\delta \dot{\boldsymbol{\xi}}
\end{array}\right\},\right. \\
& \left.+\left[\begin{array}{ccc}
\mathcal{O}_{3 \times 3} & m G & \mathcal{O}_{3 \times n} V_{c_{0}} \\
\mathcal{O}_{3 \times 3} & \mathcal{O}_{3 \times n} & \mathcal{O}_{3 \times n} \\
\mathcal{O}_{3 \times 3} & S_{1}^{T} G & -K_{e e}
\end{array}\right]\left\{\begin{array}{l}
\delta \mathbf{R}_{c} \\
\delta \boldsymbol{\theta} \\
\delta \boldsymbol{\xi}
\end{array}\right\}+\left\{\begin{array}{l}
\delta \mathbf{F} \\
\delta \mathbf{M} \\
\delta \mathbf{f}_{e}
\end{array}\right\}\right)
\end{aligned}
$$

where $M$ and $G$ matrices are as

$$
\mathbf{M}=\left[\begin{array}{ccc}
m & X_{1_{0}}^{T} & S_{1_{0}} \\
X_{1_{0}}^{T} & J_{0} & S_{2_{0}}+X_{2_{0}} \\
S_{1_{0}}^{T} & S_{2_{0}}^{T}+X_{2_{0}}^{T} & M_{e e}
\end{array}\right], \quad G=\left[\begin{array}{ccc}
0 & -g \cos \left(\theta_{0}\right) & 0 \\
-g \cos \left(\theta_{0}\right) & 0 & 0 \\
0 & g \sin \left(\theta_{0}\right) & 0
\end{array}\right] .
$$

Equation 17 can be expressed in a state-space form. The state vector and other system matrices are

$$
\left\{\begin{array}{l}
\mathbf{x}_{\mathrm{A} / \mathrm{C}}=\left\{\begin{array}{llllll}
\mathbf{V} & \boldsymbol{\omega} & \boldsymbol{\xi} & \mathbf{v} & \boldsymbol{\theta} & \mathbf{R}_{e}
\end{array}\right\}^{T} \\
\dot{\vec{x}}_{\mathrm{A} / \mathrm{C}}=A \vec{x}_{\mathrm{A} / \mathrm{C}}+B_{1} d+B \vec{u} \\
\vec{y}=C \vec{x}_{\mathrm{A} / \mathrm{C}}
\end{array}\right.
$$

where, $\vec{\theta}$ is the vector of Euler angles $\left(\left[\begin{array}{lll}\phi & \theta & \psi\end{array}\right]^{T}\right)$ and $\vec{v}$ represents structural deflection rates $(\vec{v}=\dot{\xi})$. The $B_{1}$ matrix is used to model effect of disturbances, such as gusts, on the aircraft dynamics. Elevator and aileron deflections are the two control inputs used in this work. The left and the right aileron deflect differentially for roll control and they can also be commanded individually to perform load alleviation. The total control surface deflection for each aileron is calculated by adding these two components. The proposed 2-DOF control architecture used in this work is shown in Figure 5 .

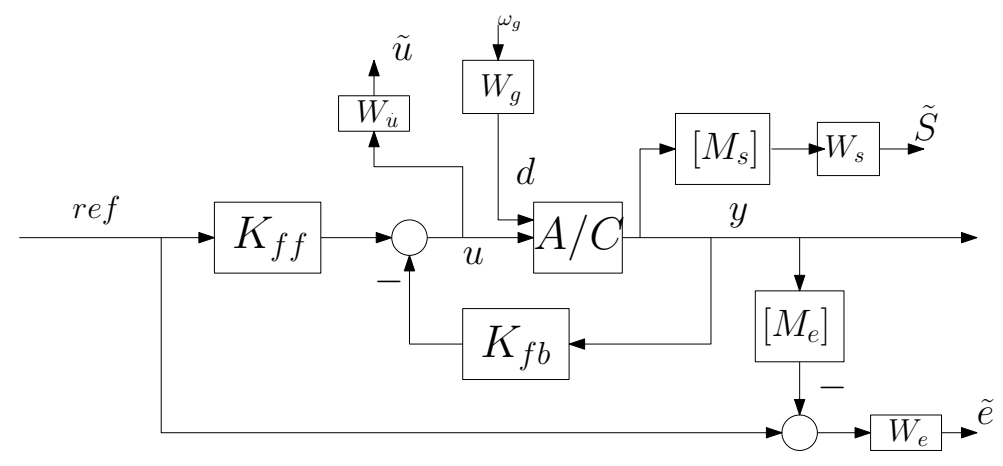

Figure 5. The proposed two DOF control architecture

where $W_{u}, W_{e}$, and $W_{s}$ are the frequency-domain weighting parameters that are employed to shape the output signals of interest $z_{\infty}=\left\{\begin{array}{ll}\rho_{e} \tilde{e} & \rho_{u_{\infty}} \tilde{u}\end{array}\right\}^{T}$ and $z_{g}=\left\{\begin{array}{cc}\rho_{s} \tilde{s} & \rho_{u_{g}} \tilde{u}\end{array}\right\}^{T}$. The following weighting functions are proposed for use in this work.

$$
\begin{aligned}
& W_{e}=\frac{s / M_{e}+\omega_{b_{e}}}{s+\omega_{b} \epsilon_{e}} \\
& W_{u}=\frac{s+\omega_{b} c_{u} / M_{u}}{\epsilon+\omega_{b c_{u}}}, \\
& W_{s}=\frac{s / M_{s}+\omega_{b_{s}}}{s+\omega_{b_{s}} \epsilon_{s}}
\end{aligned},
$$


where frequencies $\omega_{b c_{u}}, \omega_{b_{e}}$ and $\omega_{b_{s}}$ indicate the roll-off frequencies for the actuator deflections, rigid-body tracking error, and structural deformations, respectively. Finally, $\epsilon_{s}$ is used to regulate the low frequency structural deformation and $M_{u}$ is an indicator of the control surface saturation limit.

The system output is divided into two categories, while the first output vector $\left(z_{\infty}\right)$ represents the rigid-body stability and flying qualities and also the control effort. The second output $\left(z_{g}\right)$ represents the structural stability and deflections and also the control effort for regulating the structural deformations. The control system can be represented in linear fractional transformation (LFT) form, where $P$ includes the aircraft dynamics and all the frequency-based weightings (Figure 6).

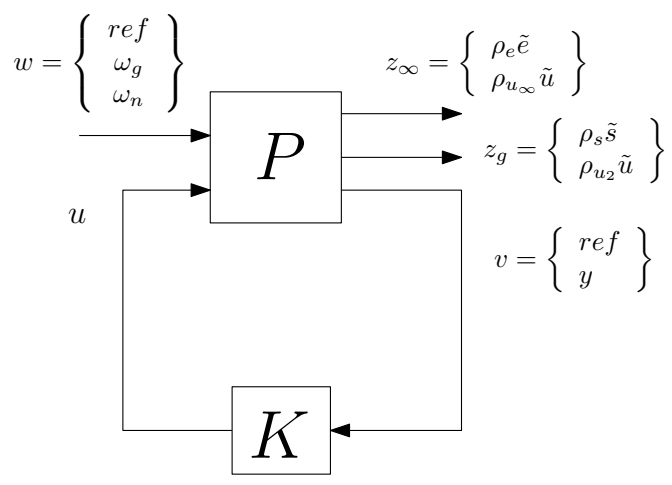

Figure 6. Control system in LFT form

The two feed-back and feed-forward controllers are combined. The gain matrix for both controllers is written as,

$$
K=\left[\begin{array}{ll}
K_{f f} & K f b
\end{array}\right]
$$

While the $\infty$-norm is better suited for stability and robustness analysis, the generalized 2-norm can better represent the output signal peak value. Therefore, a mixed-norm minimization is of interest in this work. Having all elements for control synthesis in place, the design optimization problem can be stated as,

$$
\begin{array}{cc}
\min & \left\|z_{\infty}\right\|_{\infty}+\rho_{g}\left\|z_{g}\right\|_{g} \\
\text { w.r.t. } & K
\end{array}
$$

The LMI techniques and convex optimization methods are employed to make the problem of finding the controller gains more tractable. More detailed discussion on the LMI representation of $\mathcal{H}_{\infty}$ and $\mathcal{H}_{g}$ problem can be found in the robust and optimal control literature ${ }^{19,33] 34}$ A congruency transformation is required to transform the bilinear matrix inequalities (BMI) that represent these problems into LMI problems. The resulting LMI representation for the $\mathcal{H}_{\infty}$ problem is

$$
\begin{aligned}
& \left(\begin{array}{ccc}
\left(A X+B N C_{y}\right)+\left(A X+B N C_{y}\right)^{T} & B_{1} & \left(C_{\infty} X+D_{\infty_{u}} N C_{y}\right)^{T} \\
* & -\gamma I & D_{\infty_{w}}^{T} \\
* & * & -\gamma I
\end{array}\right)<0, \\
& X>0, \\
& M C_{y}=C_{y} X \text {. }
\end{aligned}
$$

And the LMI formulation of the $\mathcal{H}_{g}$ problem is 


$$
\begin{aligned}
& \left(\begin{array}{cc}
\left(A X+B N C_{y}\right)+\left(A X+B N C_{y}\right)^{T} & B_{1} \\
* & -I
\end{array}\right)<0, \\
& \left(\begin{array}{cc}
X & \left(C_{g} X\right)^{T} \\
* & \alpha I
\end{array}\right)>0 \\
& M C_{y}=C_{y} X \text {. }
\end{aligned}
$$

In which, $A$ represents the state-matrix of the augmented plant (which includes the frequency-domain weighting functions). By using the above-mentioned LMI problems, the control design problem represented in the Equation (22) can be casted as follows,

$$
\begin{array}{cc}
\text { min. } & \gamma+\rho_{g} \alpha \\
\text { w.r.t. } & X, N \\
\text { s.t } & \text { Equations } 23,24,26 \text { and 27 }
\end{array}
$$

While $X$ and $N$ matrices are found by solving the above problem, the $M$ matrix can be found by solving Equation 25). Finally, the controller gain has the following form,

$$
K=N M^{-1} .
$$

The above LMI problems are casted in semi-definite programming form and CVXOPT (a Python-based convex optimization framework) is used to solve the problems to find the appropriate matrices and the controller gains .

\section{Optimization Formulation}

The objective of this framework is to increase the range of aircraft with high-aspect ratio wings, through the use of an active load alleviation system. The design variables are selected from all three disciplines. The wing is designed by varying the aspect ratio $(A R)$ and spanwise twist distribution $\left(\Gamma_{i}\right)$. Spar thicknesses $\left(t_{i}\right)$ are the structural sizing variables. Finally, the system-level control parameters are the relative weighting parameters $\left(\rho_{u_{\infty}}, \rho_{e}, \rho_{u_{g}}, \rho_{s}, \rho_{g}\right)$.

The main constraint which used to ensure the structural integrity is the stress distribution along the span $\left(\sigma_{\max _{i}}\right)$. The critical stress condition corresponds to the maximum stress value that the aircraft wing experiences during a discrete, $1-\cos$, gust excitation. A diagram of the proposed MDO framework is shown in Figure 7

In order to reduce the number of structural constraints, Kreisselmeier-Steinhauser (KS) function is used ${ }^{[35}$ The KS function is a differentiable envelope function with the following form:

$$
K S\left(g_{i}\right)=\left(\frac{1}{\rho}\right) \ln \left[\sum_{i=1}^{n} \exp \left(\rho g_{i}\right)\right] .
$$

The KS function satisfies the following inequality property,

$$
g_{\max } \leq K S\left(g_{i}\right) \leq g_{\max }+\frac{\ln (n)}{\rho} .
$$

A more detailed analysis on the properties of KS function and its application in optimization problems can be found in privious works ${ }^{\sqrt[35]{36}}$ Based on the above mentioned property, the stress constraints can be stated as,

$$
\begin{aligned}
& K S\left(g\left(\sigma_{i}\right)\right)<0 \\
& \text { where, } g\left(\sigma_{i}\right)=\frac{\sigma_{i}}{\sigma_{y}}-1
\end{aligned}
$$

An additional set of constraints is required to ensure the feasibility of the designed structure. These are physical constraints insure that the spar thickness is less than half of the spar diameter at different span-wise locations. 


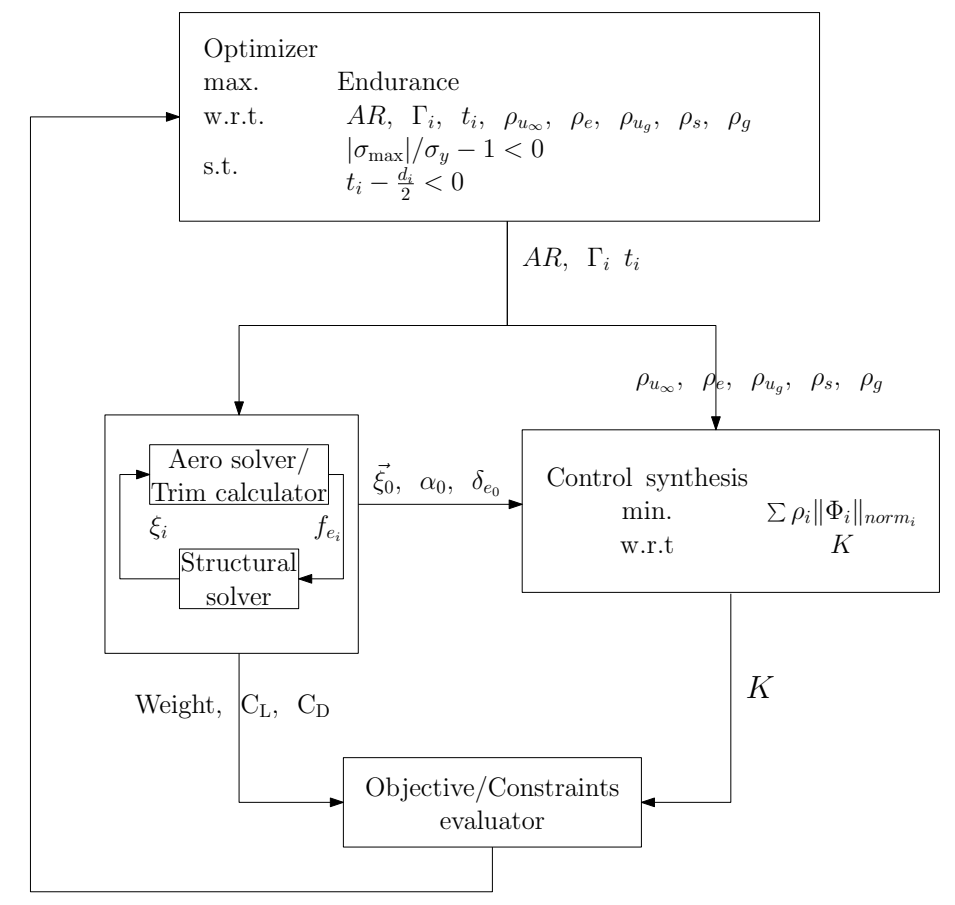

Figure 7. Proposed MDO framework for aeroservoelastic design

\section{A. Optimization Problem}

The system-level optimization can now be formulated. Spar thickness is varied at four locations along the span (root, $1 / 4$ span, $3 / 4$ span, and tip) and the intermediate values are linearly interpolated between each two adjacent design sections. The wing incidence angle is fixed at the root and its value at the tip is also a design variable. The complete system-level optimization is as follows:

$$
\begin{array}{ll}
\min & - \text { Endurance } \propto-\frac{C_{L}}{C_{D}} \ln \frac{W_{\text {initial }}}{W_{\text {emply }}} \\
\text { w.r.t } \quad A R, \quad \Gamma, \quad t_{i}, \quad \rho_{u_{\infty}}, \quad \rho_{e}, \quad \rho_{u_{g}}, \quad \rho_{s}, \quad \rho_{g} \\
\text { s.t } \quad & \quad \begin{array}{l}
K S\left(g\left(\sigma_{i}\right)\right)<0 \\
t_{i}-\frac{D_{i}}{2} \leq 0 \\
-10 \leq \Gamma_{i} \leq 10 \\
13 \leq A R \leq 20 \\
0.01 \leq \rho_{u_{\infty}} \leq 1.0 \\
0.005 \leq \rho_{e} \leq 0.5 \\
0.01 \leq \rho_{u_{g}} \leq 1.0 \\
1.0 \leq \rho_{s} \leq 30.0 \\
0.001 \leq \rho_{g} \leq 5.0
\end{array}
\end{array}
$$

A non-dimensional form of endurance is used here since the engine's specific fuel consumption was removed from the actual endurance formula. As mentioned before, the spar diameter is determined by the airfoil geometry. During the course of the optimization, $t / c$ is kept constant. This means that a variation in aspect ratio, changes the airfoil thickness and therefore the spar diameter along the span. The stress constraint is evaluated by numerically simulating the aircraft encountering a $1-$ cos vertical gust, which is given by

$$
w_{g}=\frac{\bar{w}_{g}}{2}\left(1-\cos \frac{2 \pi t}{L_{g}}\right),
$$


where $\bar{w}_{g}$ is the maximum gust velocity, and $L_{g}$ is the gust length. For our purposes, $\bar{w}_{g}$ is set to $15 \mathrm{fps}(4.575 \mathrm{~m} / \mathrm{s})$ and value of $0.5 \mathrm{~s}$ is used for $L_{g}$. The gust scale factor $\left(L_{g}\right)$ is selected so that it has the maximum excitation on the aircraft structure is achieved.

\section{B. Baseline model}

A high altitude long endurance (HALE) UAV with large aspect ratio is the target of this study. The geometry of the baseline aircraft is shown in Figure 8 and the geometrical parameters are listed in Table 1 . More detailed information of this UAV can be found in earlier work.29,37

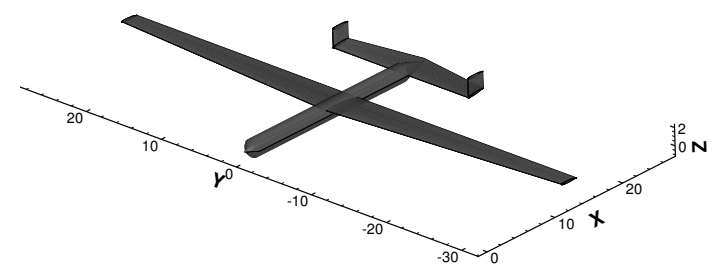

Figure 8. 3D geometry of a generic UAV with a large span
Table 1. Aircraft geometric properties

\begin{tabular}{lr}
\hline \hline Property & Value \\
\hline Fuselage length & $26.4 \mathrm{~m}$ \\
Wing span & $58.6 \mathrm{~m}$ \\
Wing area & $196 \mathrm{~m}^{2}$ \\
Wing taper ratio & 0.48 \\
HT span & $18 \mathrm{~m}$ \\
HT area & $53.5 \mathrm{~m}^{2}$ \\
HT taper ratio & 0.7 \\
VT span & $4 \mathrm{~m}$ \\
VT area & $8.9 \mathrm{~m}^{2}$ \\
VT taper ratio & 0.81 \\
\hline
\end{tabular}

A NACA 4415 airfoil is used for the wing cross-section and the chord-wise location of the spar is set to $45 \%$ of the chord. The optimization is performed for a cruise speed of $80 \mathrm{~m} / \mathrm{s}$.

\section{Results}

In this section, we present the results of the application of the controller to gust and maneuver load alleviation system . For both the gust and maneuver cases, the results are compared against the same flight conditions with no the load alleviation. The obtained results are presented in Figures 9 and 10 . 

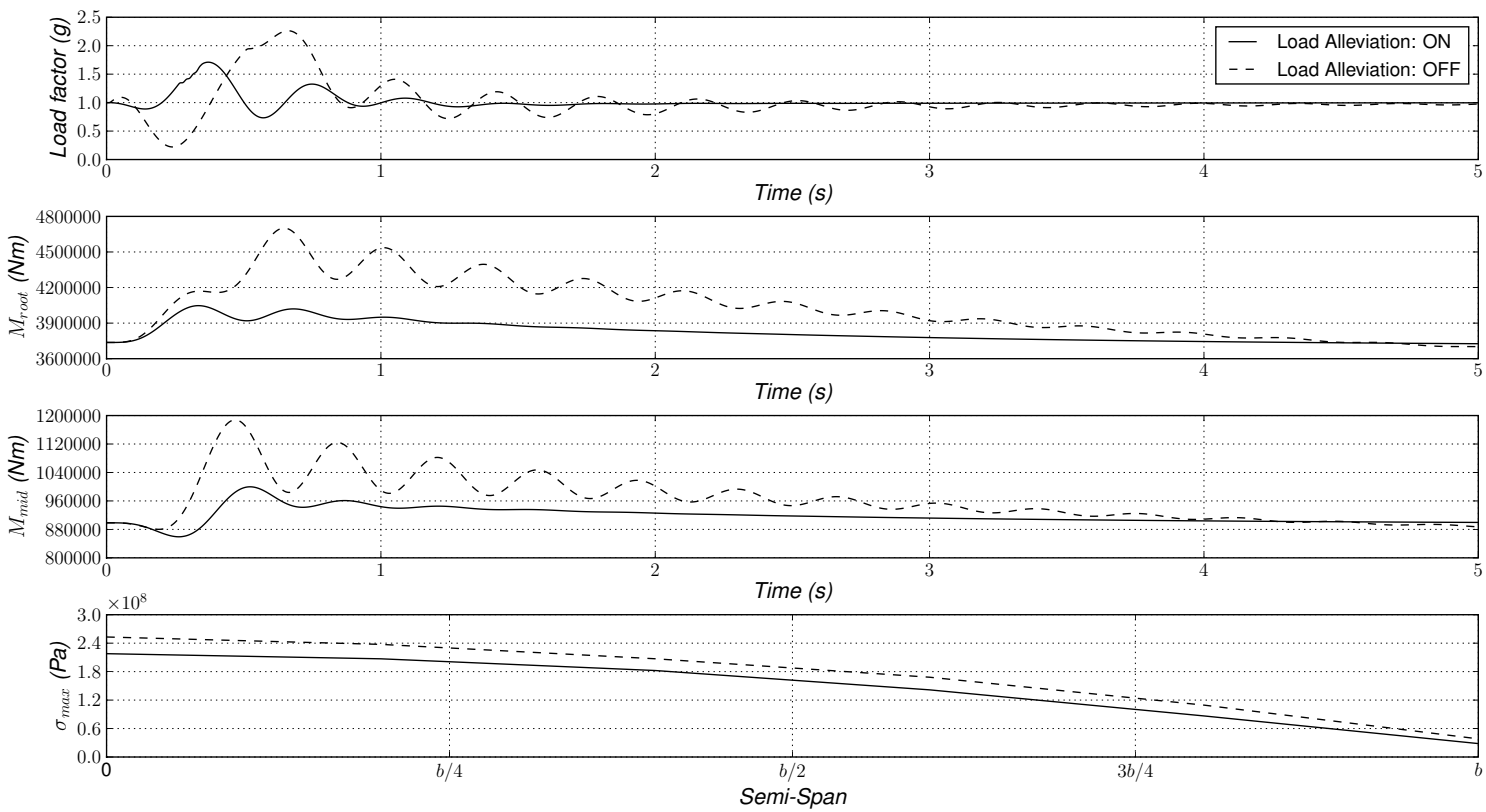

Figure 9. Gust induced load factor and maximum stress along the span
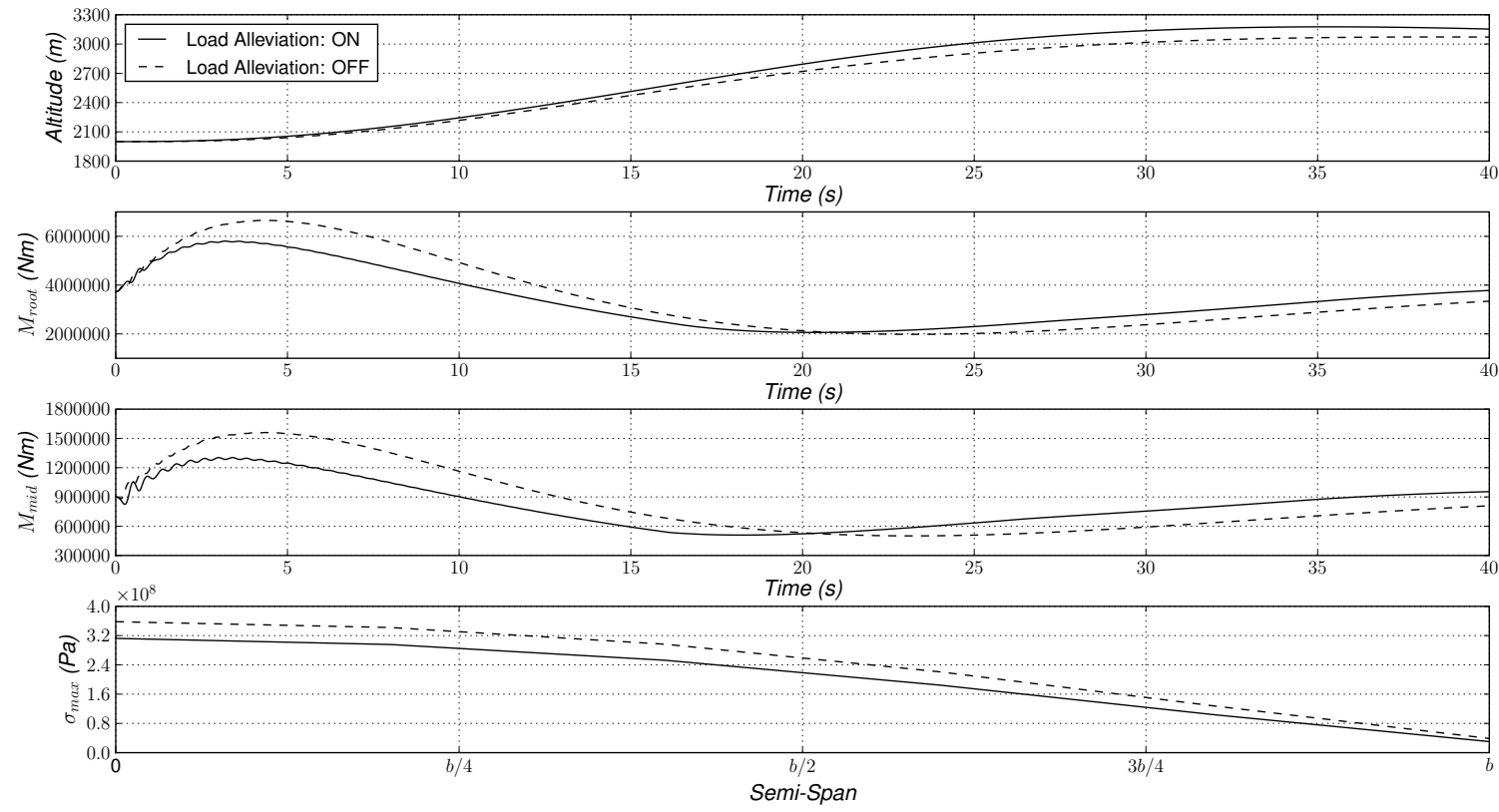

Figure 10. Altitude maneuver (climb) and maximum stress along the span

In both flight conditions, the load alleviation system has manged to significantly lower the magnitude of the maximum stress along the wing span. In the second flight condition, a $1000 \mathrm{~m}$ climb is performed by the two controllers and as it can be seen, not only is the stress level is reduced when the load alleviation system is on but the maneuver is also performed at a faster rate. Thus, the load alleviation was not achieved at the cost of lower maneuverability.

\section{A. Optimization Results}

The results of the optimization with and without the inclusion of the load alleviation system is presented are Figures 11, 12 and in Table 2. The optimization results with load alleviation show a $7.82 \%$ increase in the aircraft endurance. Although, the apste-ratio and the $L / D$ is lower when load alleviation is on but the aircraft weight is reduced by $6.55 \%$. 

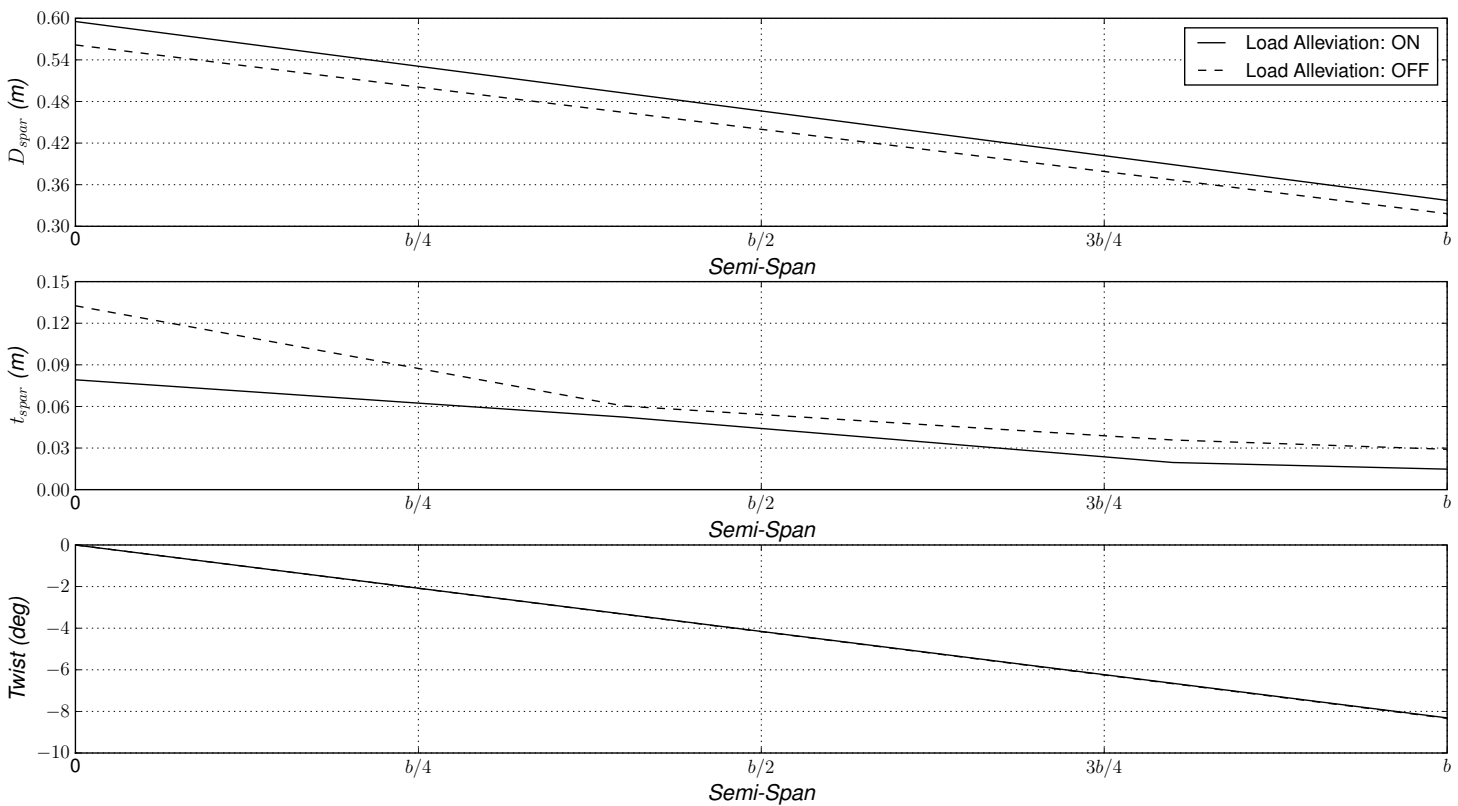

Figure 11. Design variables distribution along the span
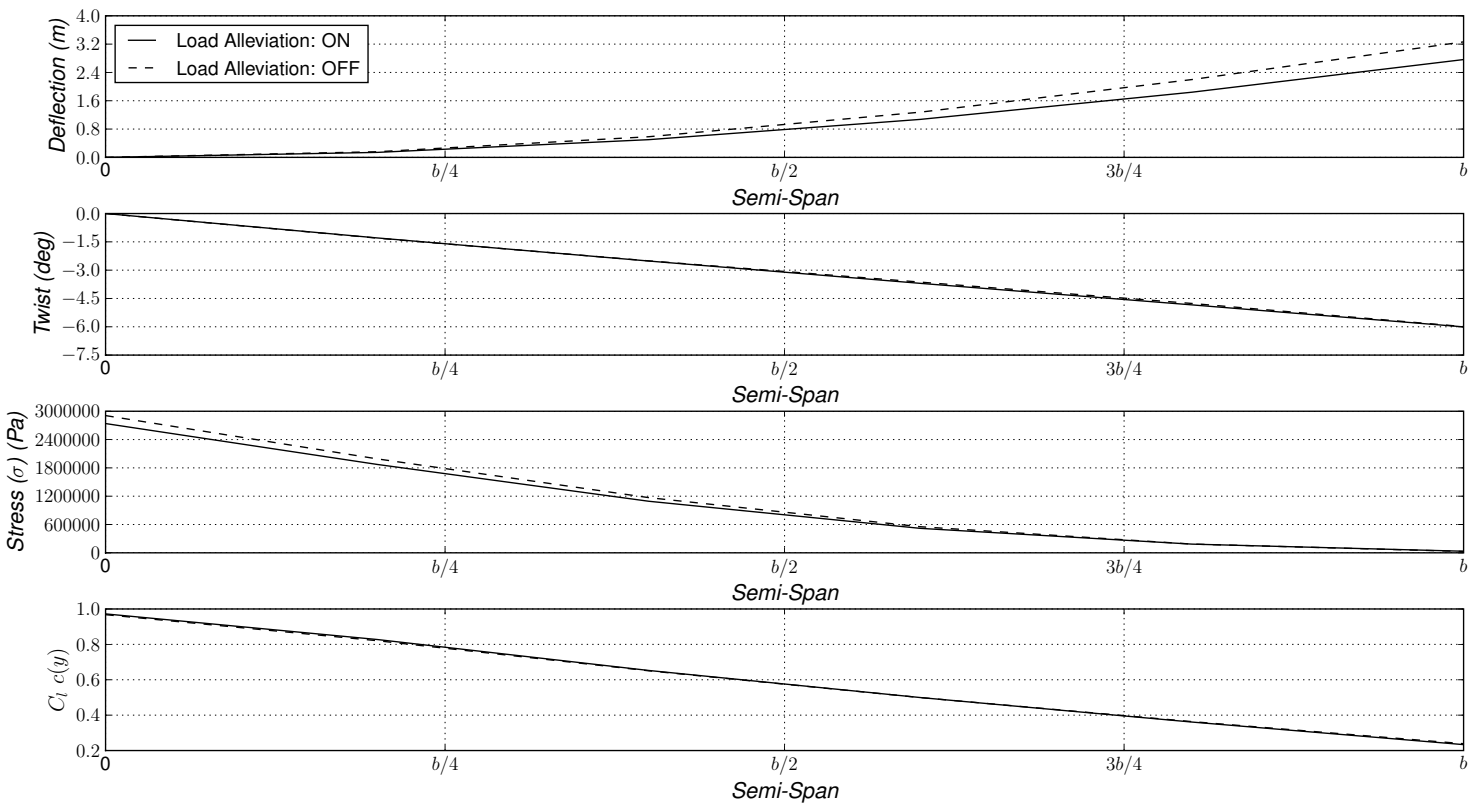

Figure 12. Wing deflection, stress, and $C_{l}$ distribution along the span (cruise condition) 
Table 2. Optimization results

\begin{tabular}{l|l|l}
\hline \hline & Load Alleviation: On & Load Alleviation: Off \\
\hline $\mathrm{AR}$ & 17.70718 & 20 \\
\hline$\rho_{u_{\infty}}$ & 0.603 & .011 \\
\hline$\rho_{e}$ & 0.3248 & 0.5 \\
\hline$\rho_{u_{g}}$ & 0.01 & 0.0101 \\
\hline$\rho_{s}$ & 1.014 & 11.501 \\
\hline$\rho_{g}$ & 4.71744 & 0.04264 \\
\hline$C_{L} / C_{D}$ & 39.25 & 40.97 \\
\hline weight (N) & 48349.94 & 51739.05 \\
\hline Endurance (Non-dim) & 42.557 & 39.4836 \\
\hline
\end{tabular}

Red values at their lower bounds

Blue values at their upper bounds

\section{Conclusions}

We integrated the control system design and time-domain techniques into aircraft multidisciplinary design optimization. In order to perform time-history analysis, the equations of motion of a very flexible aircraft that can handle large rigid-body motions and account for the structural deformations were developed. The formulation derived herein was used to establish an MDO framework that integrates control system design and aerostructural design of an aeroservoelastic aircraft at the conceptual design level. A 2-DOF mixed-norm control architecture that is capable of performing load alleviation was proposed for the design process. The control structure was specifically designed to provide the optimizer with enough freedom in designing the control gains and also the architecture is well-suited for both regulation and tracking applications. The proposed architecture was casted in LMI form where convex optimization techniques can be used to solve the control problem very efficiently. The results of applying the developed controller to the baseline aircraft have been presented and the load alleviation system was shown to be very effective in lowering the stress level without sacrificing the maneuverability. Finally, the proposed framework was linked with ALPSO to optimize the aircraft endurance while maintaining the structural stresses due to gust loads below the yield stress.

\section{References}

${ }^{1}$ Livne, E., "Integrated Aeroelastic Optimization: Status and Direction," Journal of Aircraft, Vol. 36, No. 1, 1999, pp. 122 - 145.

${ }^{2}$ Woods-Vedeler, J. and Pototzky, A., "Rolling Maneuver Load Alleviation Using Active Controls," Journal of Aircraft, Vol. 32, No. 1, 1995, pp. $68-76$.

${ }^{3}$ Suzuki, S., "Simultaneous Structure/Control Design Synthesis for Aero-Servo-Elastic System," Finite Elements in Analysis and Design, Vol. 14, No. 2 - 3, October 1993, pp. 197 - 208.

${ }^{4}$ Nam, C., Chattopadhyay, A., and Kim, Y., "Optimal Wing Planform Design for Aeroelastic Control," AIAA Journal, Vol. 38, No. 8, 2000, pp. $1455-1470$.

${ }^{5}$ Moulin, B. and Karpel, M., "Gust Loads Alleviation Using Special Control Surfaces,” Journal of Aircraft, Vol. 44, No. 1, 2007, pp. 17 - 25.

${ }^{6}$ Gaulocher, S., Roos, C., and Cumer, C., "Aircraft Load Alleviation During Maneuvers Using Optimal Control Surface Combinations," Journal of Guidance, Control, and Dynamics, Vol. 30, No. 2, 2007, pp. 591 - 600.

${ }^{7}$ Idan, M., Karpel, M., and Moulin, B., "Aeroservoelastic Interaction Between Aircraft Structural and Control Design Schemes," Journal of Guidance, Control, and Dynamics, Vol. 22, No. 4, 1999, pp. 513 - 519.

${ }^{8}$ Moulin, B., Idan, M., and Karpel, M., "Aeroservoelastic Structural and Control Optimization Using Robust Design Schemes," Journal of Guidance,Control, and Dynamics, Vol. 25, No. 1, 2002, pp. 152 - 159.

${ }^{9}$ Zink, S., Raveh, D., and Mavris, D., "Integrated Trim and Structural Design Process for Active Aeroelastic Wing Technology," Journal of Aircraft, Vol. 40, No. 3, 2003, pp. 523 - 531.

${ }^{10}$ Zink, S., Raveh, D., and Mavris, D., "Robust Structural Design of an Active Aeroelastic Wing with Maneuver Load Inaccuracies," Journal of Aircraft, Vol. 41, No. 3, 2004, pp. 585 - 593.

${ }^{11}$ Anderson, M. R. and Mason, W. H., "An MDO Approach to Control-Configured-Vehicle Design," 6th AIAA/NASA/ISSMO Symposium on Multidisciplinary Analysis and Optimization, Bellevue, WA, 1996, pp. 734 - 743

${ }^{12}$ Perez, R. E., Liu, H. H., and Behdinan, K., "Multidisciplinary Optimization Framework for Control-Configuration Integration in Aircraft Conceptual Design," Journal of Aircraft, Vol. 43, No. 6, November - December 2006, pp. 1937-1948. 
${ }^{13}$ Grossman, B., Strauch, G., Eppard, W. H., Gurdal, Z., and Haftka, R. T., "Integrated Aerodynamic/Structural Design of a Sailplane Wing," Journal of Aircraft, Vol. 25, No. 9, 1988, pp. $855-560$.

${ }^{14}$ Chittick, I. R. and Martins, J. R. R. A., “An asymmetric suboptimization approach to aerostructural optimization,” Optimization and Engineering, Vol. 10, No. 1, 2009, pp. 133 - 152.

${ }^{15}$ Noll, T. E., Ishmael, S. D., Henwood, B., Perez-Davis, M. E., Tiffany, G. C., Madura, J., Gaier, M., Brown, J. M., and Wierzbanowski, T., "Technical Findings, Lessons Learned, and Recommendations Resulting from the Helios Prototype Vehicle Mishap," Tech. Rep. 20070022260, NASA, 2007.

${ }^{16}$ Haghighat, S., Liu, H. H. T., and Martins, J. R. R. A., "Gust Load Alleviation Using Model Predictive Control for Large Aspect Ratio UAV Wings," Canadian Aeronautics and Space Institute Annual General Meeting, Ottawa, Canada, May 2009.

${ }^{17}$ Haghighat, S., Liu, H. H. T., and Martins, J. R. R. A., "Application of Model Predictive Control to Gust Loads Alleviation Systems," AIAA Atmospheric Flight Mechanics, Chicago, AIAA 2009 - 5929, 2009.

${ }^{18}$ Haghighat, S., Martins, J. R. R. A., and Liu, H. H. T., "Integrating an Active Control System with the Structural Design of a Flexible Wing Using Multidisciplinary Optimization," International Forum on Aeroelasticity and Structural Dynamics, Seattle, USA, June 2009.

${ }^{19}$ Scherer, C., Gahinet, P., and Chilali, M., "Multiobjective Output-Feedback Control via LMI Optimization," Transaction on Automatic Control, Vol. 42, No. 7, July 1997, pp. 896 - 911.

${ }^{20}$ Perez, R. E. and Behdinan, K., Swarm Intelligence: Focus on Ant and Particle Swarm Optimization, chap. Particle Swarm Optimization in Structural Design, Itech Education and Publishing, Vienna, Austria, 2007, pp. 532 - 553.

${ }^{21}$ Pendleton, E., Bessette, D., Field, P., Miller, G., and Griffin, K., "Active Aeroelastic Wing Flight Research Program: Technical Program and Model Analytical Development," Journal of Aircraft, Vol. 37, No. 4, 2000, pp. $554-561$.

${ }^{22}$ Rodden, W. P. and Love, J. R., "Equations of Motion of a Quasisteady Flight Vehicle Utilizing Restrained Static Aeroelastic Characteristics," Journal of Aircraft, Vol. 22, No. 9, 1985, pp. $802-809$.

${ }^{23}$ McLean, D. and Prasad, R. A., "A Structural Load Alleviation Control System for a Large Aircraft," Transactions of the Institute of Measurement and Control, Vol. 2, No. 1, Jan. - Mar. 1980, pp. 25 - 37.

${ }^{24}$ Aouf, N., Boulet, B., and Botez, R., "Robust Gust Load Alleviation for a Flexible Aircraft," Canadian Aeronautics and Space Journal, Vol. 46, No. 3, September 2000, pp. $131-140$.

${ }^{25}$ Waszak, M. R. and Schmidt, D. K., "Flight Dynamics of Aeroelastic Vehicles," Journal of Aircraft, Vol. 25, No. 6, 1988, pp. 563 - 571.

${ }^{26}$ Schmidt, D. K. and Raney, D. L., "Modeling and Simulation of Flexible Flight Vehicles," Journal of Guidance, Control and Navigation, Vol. 24, No. 3, 2001, pp. $539-546$.

${ }^{27}$ Meirovitch, L. and Tuzcu, I., "Integrated Approach to the Dynamics and Control of Maneuvering Flexible Aircraft," Tech. Rep. CR-2003211748, NASA, June 2003.

${ }^{28}$ Patil, M. J. and Hodges, D. H., "Flight Dynamics of Highly Flexible Flying Wings," Journal of Aircraft, Vol. 43, No. 6, 2006, pp. 1791-1798.

${ }^{29}$ Shearer, C. M. and Cesnik, C. E., "Nonlinear Flight Dynamics of Very Flexible Aircraft," Journal of Aircraft, Vol. 44, No. 5, 2007, pp. 1528 -1545 .

${ }^{30}$ Baluch, H. A. and van Tooren, M., "Modified Inertially Coupled Equations of Motion for Flexible Aircraft with Coupled Vibrations," Journal of Aircraft, Vol. 46, No. 1, 2009, pp. $107-115$.

${ }^{31}$ Karpel, M., "Procedures and Analysis for Aerservoelastic Analysis and Design," Journal of Applied Mathematics and Mechanics (ZAMM), Vol. 81, No. 9, 2001, pp. $579-592$.

${ }^{32}$ Paz, M. and Leight, W., Structural Dynamics, Theory and Computation, Kluwer Academic Publisher, 2004.

${ }^{33}$ S. Boyd, L, E. G. E. F. and Balakrishnan, V., Linear Matrix Inequalities in System and Control Theory, SIAM, 1994.

${ }^{34}$ Lee, C., Salapaka, S. M., and Voulgaris, P. G., "Two Degree of Freedom Robust Optimal Control Design using A Linear Matrix Inequality Optimization," Joint 48th IEEE Conference on Decision and Control and 28th Chinese Control Conference, 2009.

${ }^{35}$ Sobieski, J. S., "A Technique for Locationg Function Roots and for Satisfying Equality Constraints in Optimization," Structural Optimization, Vol. 4, No. 3 - 4, 1992, pp. $241-243$.

${ }^{36}$ Martins, J. R. R. A. and Poon, N. M. K., "On structural optimization using constraint aggregation," Proceedings of the VI World congress of structural and multidisciplinary optimization, 2005.

${ }^{37}$ Shearer, C. M. and Cesnik, C. E. S., "Trajectory Control for Very Flexible Aircraft," Journal of Guidance, Control, and Dynamics, Vol. 31, No. 2, 2008, pp. $340-357$. 